\title{
Survival after extracorporeal cardiopulmonary resuscitation in infants and children with heart disease
}

\author{
Titus Chan, MD, ${ }^{a}$ Ravi R. Thiagarajan, MBBS, MPH, ${ }^{\mathrm{b}}$ Deborah Frank, MD, PhD, ${ }^{a}$ and Susan L. Bratton, MD, MPH ${ }^{\mathrm{a}}$
}

Objective: We investigated survival and predictors of mortality for infants and children with heart disease treated with extracorporeal membrane oxygenation as an aid to cardiopulmonary resuscitation.

Methods: Children ( $<18$ years) with heart disease who received extracorporeal cardiopulmonary resuscitation and were reported to the Extracorporeal Life Support Organization database were evaluated. Patients were classified into one of 3 groups based on underlying cardiac physiology: single ventricle, 2 ventricles, and cardiac muscle disease. Patients with eligible procedure codes were assigned a Risk Adjustment for Congenital Heart Surgery-1 classification.

Results: Four hundred ninety-two patients were eligible for analysis, and 279 (57\%) were assigned a Risk Adjustment for Congenital Heart Surgery-1 category. Overall survival was $42 \%$. In a multivariable logistic regression analysis, significant pre-extracorporeal predictors for mortality included single-ventricle physiology (odds ratio, 1.6; 95\% confidence interval, 1.05-2.4), a history of a stage 1-type procedure (odds ratio, 2.7; 95\% confidence interval, 1.2-6.2), and extreme acidosis (arterial blood gas $\mathrm{pH}<7.01$; odds ratio, 2.2; 95\% confidence interval, 1.3-3.7). Right carotid artery cannulation was associated with decreased mortality risk (odds ratio, 0.6 ; 95\% confidence interval, 0.4-0.9). During extracorporeal support, complications, including renal injury, evidence of neurologic injury, and persistent acidosis, were associated with an increased risk of hospital mortality.

Conclusion: Use of extracorporeal membrane oxygenation as an adjunct to cardiopulmonary resuscitation resulted in hospital survival in $42 \%$ of infants and children with heart disease. Underlying cardiac physiology and associated cardiac surgical procedures influenced mortality, as did pre-extracorporeal resuscitation status and extracorporeal membrane oxygenation-associated complications.

From the Division of Pediatric Critical Care, Department of Pediatrics, ${ }^{\text {a }}$ Primary Children's Medical Center, University of Utah, Salt Lake City, Utah and the Division of Cardiology, Children's Hospital Boston, and the Department of Pediatrics Harvard Medical School, ${ }^{\mathrm{b}}$ Boston, Mass.

Received for publication Nov 8, 2007; revisions received Feb 6, 2008; accepted for publication March 2, 2008.

Address for reprints: Titus Chan, MD, Department of Pediatrics, Division of Critical Care Medicine, University of Utah, PO Box 581289, Salt Lake City, UT 841581289 (E-mail: tituschan@gmail.com).

J Thorac Cardiovasc Surg 2008;136:984-92 0022-5223/\$34.00

Copyright $(\underset{2008}{ } 20 y$ The American Association for Thoracic Surgery

doi:10.1016/j.jtcvs.2008.03.007
$\mathrm{T}$ The mainstay for the resuscitation of children after cardiopulmonary arrest has been conventional cardiopulmonary resuscitation (CPR). However, survival after CPR has variable results, with recent reports of hospital survival ranging between $12 \%$ and $41 \% .^{1,2}$ There has been an emerging interest in the use of extracorporeal membrane oxygenation (ECMO) as a rescue technique (ECPR) for patients in whom conventional CPR has not re-established a perfusing rhythm and adequate circulation. del Nido and colleagues ${ }^{3}$ were the first to describe the use of ECPR in infants and children who had previous congenital heart surgery and demonstrated that the use of ECPR promoted survival in these patients. Patients with congenital heart disease who have undergone cardiac surgery often have a reversible cause for their cardiac arrest in the postoperative period and might benefit from ECMO support if the underlying reason for the cardiac arrest is adequately treated. Infants and children with congenital heart disease are the largest proportion of patients treated with ECPR. ${ }^{4}$

Most reports examining ECPR have been single-institution analyses and have mainly examined periarrest circumstances, such as duration of chest compressions, on hospital mortality. ${ }^{4,5}$ There has been little research examining the effect of specific cardiac physiology and recent congenital heart surgery on the outcomes of ECMO 


\section{Abbreviations and Acronyms \\ $\mathrm{CPR}=$ cardiopulmonary resuscitation \\ $\mathrm{CPT}=$ Current Procedural Terminology \\ ECMO = extracorporeal membrane oxygenation \\ ECPR = extracorporeal membrane oxygenation to aid cardiopulmonary resuscitation \\ ELSO = Extracorporeal Life Support Registry \\ ICD-9 = International Classification of Diseases-9th Edition \\ IQR = interquartile range \\ RACHS-1 = Risk Adjustment for Congenital Heart Surgery-1}

used as ECPR. Because ECMO programs and therapy are expensive and considerable institutional resources are needed to provide rapid deployment of ECMO for ECPR, it is important to understand the factors that influence mortality in this patient group that heavily uses ECPR.

In this study we used data from children with heart disease who received ECPR as reported to the Extracorporeal Life Support Registry (ELSO). This was a secondary analysis from a larger dataset examining ECPR outcomes in all children, irrespective of heart disease status. ${ }^{6}$ Our goal was to determine whether outcomes for these children were associated with patient demographics, pre-ECMO management, type of cardiac disease, and ECMO support variables.

\section{Materials and Methods}

The ELSO registry contains voluntarily submitted data on infants, children, and adults who have undergone extracorporeal cardiopulmonary support at one of 140 international contributing ECMO centers. Each ECMO center submits data through standardized ECMO data sheets that contain patient demographics, primary and secondary diagnoses and procedures, ECMO techniques and complications, and patient hospital survival. ELSO defines ECPR as "Extracorporeal Cardiopulmonary Resuscitation, in which ECLS (extracorporeal life support) was used as part of the initial resuscitation from cardiac arrest. Patients who are hemodynamically unstable and placed on ECLS emergently without a cardiac arrest are not considered E-CPR." Patients who used ECMO to aid CPR are specifically coded and can be identified from the ELSO data registry.

We selected patients with a primary or secondary diagnosis of cardiac disease from patients who received ECPR during 19922005 and were less than 18 years of age by using their primary and secondary diagnosis (International Classification of Diseases9th Edition [ICD-9] $]^{7}$ ) and procedure codes (Current Procedural Terminology $\left.[\mathrm{CPT}]^{8}\right)$. Subjects were divided into one of 3 diagnostic categories based on cardiac disease-single-ventricle lesions, 2ventricle lesions including primary pulmonary hypertension, and cardiac muscle disease (cardiomyopathy or myocarditis) — by using ICD-9 diagnosis codes and based on their physiology at the time of ECPR use. Heart transplantation was classified as muscle disease, and arrhythmias without other structural anomalies reported were also classified as cardiac muscle disease. Patients were classified by 3 independent authors (TC, SLB, RRT), and disagreements were resolved by a fourth opinion (DF). Any patient in whom consensus on a diagnostic category could not be achieved was excluded from the analysis $(\mathrm{n}=10)$.

Additionally, patients who had undergone congenital heart surgery procedures were placed into 6 procedural complexity-based categories by using the Risk Adjustment for Congenital Heart Surgery-1 (RACHS-1) method. ${ }^{9}$ The RACHS-1 is a method of risk adjustment for in-hospital mortality that accounts for surgical complexity. Increasing RACHS-1 categories are associated with increasingly complex surgical procedures and increasing risk of inhospital mortality. RACHS-1 categories were assigned based on available CPT codes. The RACHS-1 categories were assigned based on the highest-risk procedure reported if multiple CPT codes were available.

Information regarding patient demographics, pre-ECMO support modalities, pre-ECMO blood gas data, ECMO techniques and complications, and survival to hospital discharge was evaluated. Low $\mathrm{pH}$ before ECMO has previously been reported to be associated with mortality. ${ }^{6}$ Blood gas $\mathrm{pH}$ was trichotomized based on $\mathrm{pH}$ data of subjects who died, with the "lowest" $\mathrm{pH}$ being less than the 25th quartile, the "medium" $\mathrm{pH}$ determined by the 25 th quartile, and the median value and the "higher" $\mathrm{pH}$ being greater than the median value. Modes of ECMO were classified into venovenous, venoarterial, or combination groups if an additional drainage or infusion site was used. Arterial cannulation was classified into one of 3 groups: right carotid, aorta, and "other," which included femoral artery and left carotid artery cannulation.

ECMO complications were classified by using complication codes created by the ELSO registry for reporting complication-related information to the registry. These included complications classified by systems, as well as subgroupings within each systems group. The complications were grouped as follows: (1) brain injury (seizures [clinical or electroencephalographic evidence of seizures] and radiologic evidence of central nervous system injury [ultrasonographic or computed tomographic scan evidence of hemorrhage or infarction]); (2) renal injury (serum creatinine value $>1.5 \mathrm{mg} / \mathrm{dL}$ and dialysis use [hemodialysis or continuous arteriovenous hemodialysis]); (3) bloodstream infection defined as blood culture-proved infection; (4) ECMO support (arterial blood $\mathrm{pH}<7.2$ on ECMO, mechanical failure of ECMO circuit, and cannulation site or surgical site bleeding); (5) respiratory complications (pulmonary hemorrhage and pneumothorax requiring treatment); (6) cardiac complications (arrhythmias during ECMO support that required treatment and CPR during ECMO support); and (7) gastrointestinal complications (gastrointestinal hemorrhage and hyperbilirubinemia defined as serum direct bilirubin level $>2 \mathrm{mg} / \mathrm{dL}$, indirect bilirubin level $>13 \mathrm{mg}$ / $\mathrm{dL}$, or total bilirubin level $>15 \mathrm{mg} / \mathrm{dL}$ ).

Only 1 ECMO run for each patient was included in the analysis. For patients who had more than 1 ECMO run $(n=12)$, the first ECMO run was included in the analysis. Demographics, preECMO data, ECMO data, and ECMO complications were compared between survivors and nonsurvivors. The $\chi^{2}$ test or the Fisher test were used for dichotomous data. The Student $t$ test and analysis of variance tests were used to compare normally distributed continuous data, whereas the Wilcoxon-Mann-Whitney test and the KruskalWallis analysis of variance were used for comparison of skewed 
data. Data are presented as mean values with the standard deviation or median values with the interquartile range (IQR; 25th and 75th quartiles).

To determine whether cardiac diagnostic categories were independently associated with survival after ECPR, a multivariable logistic regression model was developed. Factors associated with hospital death were evaluated for inclusion in the multivariable models by using the cardiac diagnostic groups. A $P$ value of less than .10 in the bivariable analysis was required for consideration of inclusion in the multivariable model. An iterative forward selection process was used for variable entry, with the criterion for inclusion being a $P$ value of less than .05 , and the criterion for exclusion being a $P$ value of greater than .10. Patients with 2 ventricles served as the reference group. The majority of variables were included as dichotomous variables, whereas other variables were trichotomized into ordinal variables by using one category as a referent. For arterial cannulation, aortic cannulation was used as the reference group, with right carotid cannulation and all other sites as comparators. Overfitting was avoided by limiting the number of variables to N/ 10 in each model. Another model using RACHS-1 categories rather than the cardiac diagnosis groups was also developed for the patients in whom a RACHS-1 classification was possible. Finally, a model was constructed examining ECMO complications, irrespective of anatomic classification or RACHS-1 categories, for factors associated with death after ECPR initiation. Survival was defined as survival to hospital discharge to either home or another facility. A c-statistic was computed for each model as a measure of goodness of fit. Stata 9.2 software (Stata Corp LP, College Station, Tex) was used for the analysis.

\section{Results}

\section{Study Population}

Six hundred eighty-two patients underwent ECPR from 1992-2005. Of these, 514 ECMO runs were associated with underlying heart disease. Of these ECMO runs, 12 were excluded as being repeat ECMO runs. Among the patients who underwent more than 1 ECMO run, mortality was $50 \%$. There were 3 patients with single-ventricle lesions ( 2 survived), 7 patients with 2-ventricle lesions (4 survived), and 2 patients with muscle disease (none survived). An additional 10 patients were excluded because of lack of consensus regarding diagnostic classification, leaving 492 patients eligible for analysis (78\% of all ECPR pediatric patients). From this cohort, 285 patients had cardiac surgical procedure codes, and 279 patients had sufficient data in the registry for RACHS- 1 category assignment (57\% of all cardiac patients). The median age of the study population was 80 days (IQR, 12-452 days), and the median weight was $4.3 \mathrm{~kg}$ (IQR, $3.2-9.5 \mathrm{~kg}$; Table 1). The distribution of diagnostic classifications included single-ventricle lesion $(\mathrm{n}=196,40 \%)$, 2 -ventricle lesion $(\mathrm{n}=186,38 \%)$, and muscle disease $(\mathrm{n}=$ $110,22 \%)$. The RACHS-1 classification included category $1(\mathrm{n}=5,2 \%)$, category $2(\mathrm{n}=34,12 \%)$, category $3(\mathrm{n}=$ $134,48 \%)$, category $4(\mathrm{n}=28,10 \%)$, and category $6(\mathrm{n}=$ $78,28 \%$ ). No RACHS-1 category 5 procedures were assigned in the study sample.

\section{Demographic Information}

The total number of ECPR cases used in cardiac patients increased from 57 between the years 1992-1999 to 256 during 2002-2005. Patients with single-ventricle lesions (median age, 16 days) were younger than patients with 2-ventricle lesions (median age, 105 days), with $60 \%$ of the single-ventricle group being less than 1 month of age. Conversely, patients with muscle disease (median age, 710 days) were older than patients in both the single- and 2-ventricle groups. Fifty-five percent of patients with muscle disease were older than 1 year. Patients with muscle disease had equal sex distribution, whereas a larger proportion of male children were present in both the single-ventricle $(60 \%)$ and 2-ventricle (64\%) groups. There was also an equal distribution of patients with muscle disease into white and nonwhite categories, whereas the single-ventricle $(62 \%)$ and 2-ventricle (65\%) groups had a higher proportion of white patients.

Patients who survived to hospital discharge or transfer are compared with those who died in Table 1. Overall survival was $42 \%$. Patients with single-ventricle lesions had a lower survival rate compared with that of patients with 2-ventricle lesions ( $P=.02, \chi^{2}$ test), whereas there was no difference in survival between those with 2-ventricle lesions and those with muscle disease $\left(P=.6, \chi^{2}\right.$ test) or between those with single-ventricle lesions and those with muscle disease $(P=$ $.1, \chi^{2}$ test). As expected, higher RACHS- 1 categories were associated with lower rates of survival among subjects for whom a RACHS- 1 category could be assigned $(P=.003$, trend test). There were no differences in age, weight, or year of ECMO treatment between survivors and nonsurvivors. There was a nonsignificant trend toward lower survival among nonwhite patients compared with white patients (37\% vs $46 \%, P=.07)$.

\section{Pre-ECMO and ECMO Support Information}

The ELSO data include information on ventilator settings and respiratory support information immediately before ECPR. This includes the worst arterial blood gas recorded in the 6 hours before the initiation of ECMO. There were no differences in ventilator settings and use of other respiratory modalities between survivors and nonsurvivors, with the exception of the mean airway pressure, which was higher among survivors (Table 2). Survivors tended to have a shorter time period of mechanical ventilation before the initiation of ECMO compared with nonsurvivors. Median pre-ECMO blood gas values were similar between survivors and nonsurvivors. When blood gas $\mathrm{pH}$ measurements were trichotomized, there was a higher proportion of nonsurvivors with a pH of less than 7.01 before ECPR $(P=.001$, individual $\chi^{2}$ test).

Table 2 also includes immediate ECMO management features. The vast majority of survivors and nonsurvivors were treated with venoarterial ECMO (93\% vs $91 \%$, respectively), with a small proportion being managed with a combination 
TABLE 1. Selected demographic features of subjects comparing survivors and nonsurvivors

\begin{tabular}{|c|c|c|c|}
\hline Characteristic & Survivors $(n=208$ ) & Nonsurvivors $(n=284)$ & Statistical test, $P$ value \\
\hline Cardiac diagnosis, n (\%) & & & Fisher exact test, $.05 \dagger$ \\
\hline One ventricle & $70(36 \%)$ & $126(64 \%)$ & \\
\hline Two ventricles/ pulmonary hypertension & $89(48 \%)$ & $97(52 \%)$ & \\
\hline Muscle disease & $49(45 \%)$ & $61(55 \%)$ & \\
\hline Age, d (median [IOR]) & $82(12-442)$ & $77(14-1092)$ & Wilcoxon-Mann-Whitney, .6 \\
\hline Weight, kg (median [IQR]) & $4.4(3.2-8.8)$ & $4.3(3.1-10)$ & Wilcoxon-Mann-Whitney, .6 \\
\hline Sex, $n(\%)$ & & & $\chi^{2}$ test, $.8 \dagger$ \\
\hline Male & $124(43 \%)$ & $166(57 \%)$ & \\
\hline Female & $83(41 \%)$ & $117(59 \%)$ & \\
\hline Missing & $1(50 \%)$ & $1(50 \%)$ & \\
\hline Race, n (\%) & & & $\chi^{2}, .07$ \\
\hline White & $135(46 \%)$ & $161(54 \%)$ & \\
\hline Other & $73(37 \%)$ & $123(63 \%)$ & \\
\hline ECPR year, $\mathrm{n}(\%)$ & & & $\chi^{2}, .5 \dagger$ \\
\hline 1992-1999 & $26(46 \%)$ & $31(54 \%)$ & \\
\hline $2000-2002$ & $70(39 \%)$ & $109(61 \%)$ & \\
\hline 2003-2005 & $112(44 \%)$ & $144(56 \%)$ & \\
\hline RACHS-1, ${ }^{*} \mathrm{n}(\%)$ & & & Trend test, $.003 \dagger$ \\
\hline 1 & $2(40 \%)$ & $3(60 \%)$ & \\
\hline 2 & $18(53 \%)$ & $16(47 \%)$ & \\
\hline 3 & $63(47 \%)$ & $71(53 \%)$ & \\
\hline 4 & $10(36 \%)$ & $18(64 \%)$ & \\
\hline 5 & 0 & 0 & \\
\hline 6 & $22(28 \%)$ & $56(72 \%)$ & \\
\hline
\end{tabular}

IQR, Interquartile range; ECPR, extracorporeal membrane oxygenation to aid cardiopulmonary resuscitation; $R A C H S$-1, Risk Adjustment for Congenital Heart Surgery-1. ${ }^{*} \mathrm{n}=279$. †Denotes global test for significance.

mode or venovenous ECMO. Although there was no difference in mortality among all sites of arterial cannulation, patients who underwent right carotid cannulation had a lower mortality rate when compared with those undergoing aortic cannulation ( $52 \%$ vs $62 \%, P=.03$ ). There was no difference in starting flow rate on ECMO or hours on ECMO between survivors and nonsurvivors.

\section{ECMO Complications}

Surviving patients had a longer hospital length of stay compared with those who died. The median hospital length of stay was 37 days (IQR, 22-62 days) versus 14 days (IQR, 6-28 days) for survivors and nonsurvivors $(P<.001$, Wilcoxon-Mann-Whitney test). A number of ECMO complications were associated with increased mortality (Table 3). Radiologic evidence of central nervous system injury, renal injury (defined as a serum creatinine level $>1.5 \mathrm{mg} / \mathrm{dL}$ ), use of renal replacement therapy, arterial blood gas $\mathrm{pH}$ of less than 7.2 during ECMO, pulmonary and gastrointestinal hemorrhage, hyperbilirubinemia, and requirement for CPR during ECMO were all associated with increased mortality, whereas the presence of seizures, bloodstream infections, pneumothorax, mechanical failure of the ECMO pump, bleeding from the cannulation site, and arrhythmias during ECMO were not associated with in-hospital death.
ECMO can be used as a bridge to heart transplantation. Thirty patients in this study received a heart or heart-lung transplantation, and patients with muscle disease comprised the majority of this subgroup (80\%). Among transplant recipients, $17(57 \%)$ children survived.

\section{Multivariable Analysis of Factors Associated With Mortality}

Two separate multivariable models were constructed to determine pre-ECMO variables associated with death. One model included pre-ECMO variables and cardiac diagnostic classification, whereas another used RACHS-1 categories and pre-ECMO variables. These models are presented in Table 4. Both models found cannulation of the right carotid artery to be associated with lower mortality. In the model based on cardiac diagnosis classification, an arterial blood gas $\mathrm{pH}$ of less than 7.01 increased the odds of mortality (odds ratio, 2.2; 95\% confidence interval, 1.3-3.7) adjusted for cardiac diagnosis and cannulation site. Patients with single-ventricle lesions had increased odds of mortality compared with children with 2-ventricle lesions or pulmonary hypertension (odds ratio, 1.6; 95\% confidence interval, 1.05-2.4; singlevariable $P=.03$ ) adjusted for both cannulation site and pH. Similarly, children with RACHS-1 category 6 procedures had increased odds of mortality compared with patients 
TABLE 2. Pre-ECMO support and initial ECMO features

\begin{tabular}{|c|c|c|c|}
\hline Features & Survivors $(n=208$ ) & Nonsurvivors $(\mathrm{n}=\mathbf{2 8 4})$ & Statistical test, $\boldsymbol{P}$ value \\
\hline Ventilatory support, ${ }^{*} \mathrm{~mm} \mathrm{Hg}$ (median [IOR] & & & Wilcoxon-Mann-Whitney \\
\hline PIP & $25(22-34)$ & $25(22-30)$ & .3 \\
\hline PEEP & $5(4-7)$ & $5(4-5)$ & .2 \\
\hline MAP & $11(9-16)$ & $10(8-12)$ & .04 \\
\hline Arterial blood gas* (median [IOR]) & & & Wilcoxon-Mann-Whitney \\
\hline $\mathrm{PaCO}_{2}(\mathrm{~mm} \mathrm{Hg})$ & $48(35-64)$ & $46(36-68)$ & .9 \\
\hline $\mathrm{PaO}_{2}(\mathrm{~mm} \mathrm{Hg})$ & $46(32-69)$ & $40(27-68)$ & .1 \\
\hline $\mathrm{pH}$ & $7.26(7.09-7.38)$ & $7.21(7.01-7.39)$ & .1 \\
\hline Categorized arterial blood gas pH (n [\%]) & & & $\chi^{2}, .03 \dagger$ \\
\hline $\mathrm{pH}<7.01$ & $26(30 \%)$ & $60(70 \%)$ & \\
\hline $\mathrm{pH} 7.01-7.21$ & $40(41 \%)$ & $58(59 \%)$ & \\
\hline $\mathrm{pH}>7.21$ & $142(46 \%)$ & $166(54 \%)$ & \\
\hline \multicolumn{4}{|l|}{ Pre-ECMO therapies (n [\%]) } \\
\hline iNO & $18(40 \%)$ & $27(60 \%)$ & $\chi^{2}, .7$ \\
\hline HFOV & $4(57 \%)$ & $3(43 \%)$ & Fisher exact test, .5 \\
\hline Surfactant & $1(50 \%)$ & $1(50 \%)$ & Fisher exact test, 1 \\
\hline Time from intubation to ECMO, $\mathrm{h}$ (median [IQR]) & $12(2-60)$ & $20(3-97)$ & Wilcoxon-Mann-Whitney, .08 \\
\hline Mode of ECMO, $\mathrm{n}(\%)$ & & & Fisher-Freeman-Halton, $.3 \dagger$ \\
\hline Venoarterial & $193(43 \%)$ & $257(57 \%)$ & \\
\hline Venovenous & 0 & $3(100 \%)$ & \\
\hline Combination & $14(37 \%)$ & $24(63 \%)$ & \\
\hline Missing & $1(100 \%)$ & 0 & \\
\hline Arterial cannulation site, $\mathrm{n}(\%)$ & & & Fisher exact test, $.06 \dagger$ \\
\hline Right carotid artery & $109(48 \%)$ & $116(52 \%)$ & \\
\hline Aorta & $82(38 \%)$ & $134(62 \%)$ & \\
\hline Other & $13(39 \%)$ & $20(61 \%)$ & \\
\hline Venovenous & 0 & $3(100 \%)$ & \\
\hline Missing & $4(27 \%)$ & $11(73 \%)$ & \\
\hline Starting flow, $\mathrm{mL} \cdot \mathrm{kg}^{-1} \cdot \min ^{-1}$ (median [IOR]) & $100(83-124)$ & $107(84-135)$ & Wilcoxon-Mann-Whitney, .3 \\
\hline Hours on ECMO (median [IQR]) & $87(51-137)$ & $87(37-171)$ & Wilcoxon-Mann-Whitney, .9 \\
\hline
\end{tabular}

with RACHS- 1 category 1 or 2 procedures (odds ratio, 2.6; 95\% confidence interval, 1.2-5.6) adjusted for cannulation site. Blood $\mathrm{pH}$ before ECPR was not associated with mortality in this model, which might be due to a smaller sample size $(\mathrm{n}=277)$. When $\mathrm{pH}$ was retained in the model, the adjusted odds ratio for mortality of RACHS-1 category 6 procedures compared with categories 1 or 2 was 2.7 (95\% confidence interval, 1.2-6.2).

In a third model examining ECMO complications associated with death, radiologic evidence of central nervous system injury, renal injury, and an arterial blood gas $\mathrm{pH}$ of less than 7.2 during ECMO were associated with an increased risk of death. Duration of ECMO was included in the model because longer periods of ECMO exposed the patients to a greater duration for ECMO-associated complications. Use of renal replacement therapy during ECMO, pulmonary hemorrhage, and CPR during ECMO were not independently associated with increased mortality in the multivariable model. Gastrointestinal bleeding was removed from the model be- cause it predicted mortality perfectly. Additionally, hyperbilirubinemia tended to be associated with mortality (odds ratio, 3.5; 95\% confidence interval, 0.99-12.6; $P=.051$ ).

\section{Discussion}

In this analysis children with heart disease who underwent ECMO as an aid to CPR had an overall survival rate of $42 \%$. Patients with single-ventricle lesions and those whose surgical procedures were assigned a higher surgical complexity category (RACHS-1 category 6) had higher odds of mortality compared with those undergoing less complex repairs. Severe acidosis before being started on ECMO was also associated with increased mortality, whereas right carotid artery cannulation was associated with decreased mortality odds. During ECMO, persistent acidosis, renal injury, and central nervous system injury on imaging studies independently increased the odds of hospital mortality.

The survival rate after cardiac arrest in children varies significantly among different studies and ranges from $12 \%^{1}$ to 
TABLE 3. ECMO complications and hospital survival

\begin{tabular}{|c|c|c|c|}
\hline Characteristic & Survivors $(n=208), n(\%)$ & Nonsurvivors ( $n=284), n(\%)$ & Statistical test, $P$ value \\
\hline Neurologic, $\mathrm{n}(\%)$ & & & $\chi^{2}$ \\
\hline Seizures & $25(42 \%)$ & $34(58 \%)$ & 1 \\
\hline Radiologic evidence of CNS injury & $10(19 \%)$ & $42(81 \%)$ & $<.001$ \\
\hline Renal, $\mathrm{n}(\%)$ & & & $\chi^{2}$ \\
\hline Creatinine $>1.5 \mathrm{mg} / \mathrm{dL}$ & $24(27 \%)$ & $64(73 \%)$ & .002 \\
\hline Renal replacement therapy & $11(26 \%)$ & $32(74 \%)$ & .02 \\
\hline Bloodstream infection, $\mathrm{n}(\%)$ & $14(33 \%)$ & $28(67 \%)$ & $\chi^{2}, .2$ \\
\hline \multicolumn{4}{|l|}{ ECMO support, $\mathrm{n}(\%)$} \\
\hline $\mathrm{pH}<7.2$ on ECMO & $9(18 \%)$ & $40(82 \%)$ & $<.001$ \\
\hline Mechanical failure & $19(33 \%)$ & $39(67 \%)$ & .1 \\
\hline Cannula bleeding & $60(40 \%)$ & $91(60 \%)$ & .4 \\
\hline \multicolumn{4}{|l|}{ Respiratory, n (\%) } \\
\hline Pulmonary hemorrhage & $9(24 \%)$ & $28(76 \%)$ & $\chi^{2}, .02$ \\
\hline Pneumothorax & 0 & $5(100 \%)$ & Fisher exact test, .08 \\
\hline \multicolumn{4}{|l|}{ Gastrointestinal, n (\%) } \\
\hline Gastrointestinal bleed & 0 & $7(100 \%)$ & Fisher exact test, .02 \\
\hline Hyperbilirubinemia & $3(12 \%)$ & $22(88 \%)$ & $\chi^{2}, .002$ \\
\hline Cardiac, n (\%) & & & $\chi^{2}$ \\
\hline Arrhythmias on ECMO & $33(36 \%)$ & $59(64 \%)$ & .2 \\
\hline CPR on ECMO & $7(24 \%)$ & $22(76 \%)$ & .04 \\
\hline
\end{tabular}

ECMO, Extracorporeal membrane oxygenation; CNS, central nervous system; CPR, cardiopulmonary resuscitation.

$41 \% .^{2}$ In pediatric populations that do not include postoperative cardiac patients, the hospital discharge rate after cardiac arrest has been reported to be as low as $12 \%$ to $16 \% .{ }^{1,10}$ Conversely, in patients with heart disease, survival rates after cardiac arrest without the use of ECMO are between $38 \%{ }^{11}$ and $41 \% .^{2}$ The overall hospital survival for pediatric patients in the current study is comparable with that in the latter studies. Two other studies examining ECPR in children have found similar hospital survival rates for children with heart disease. ${ }^{4,12}$ However, the population of patients in this analysis and the other reports had failed conventional resuscitative techniques and likely faced high mortality in the absence of ECPR. Thus these data suggest that a subset of children with cardiac disease and persistent cardiac arrest might have a reversible process, and their survival might be improved by use of short-term mechanical circulatory support after failure to respond to conventional CPR therapies.

Although the ELSO database does not include information regarding neurologic status at hospital discharge and is a limitation to our study, 2 case series of patients undergoing ECPR suggest that the majority of patients who survive to hospital discharge have a favorable outcome. ${ }^{4,12}$ In the series by Morris and colleagues, ${ }^{4}$ half of survivors older than 2 months with follow-up had no decrement in their Pediatric Cerebral or Overall Performance Category. In a series by Alsoufi and associates, ${ }^{12} 86 \%$ of children with cardiac disease who survived had a grossly intact neurologic status.

In our study, patients with single-ventricle lesions had lower survival rates than patients with 2-ventricle lesions.
In contrast, a recent case series of patients undergoing ECPR found patients with single-ventricle lesions to have a higher survival rate $(47 \%)$ than patients with 2 -ventricle lesions $(27 \%) .^{12}$ This discrepancy might be due to a smaller sample size ( 80 patients) in the case series or might be due to differences in ECMO experience or practice style related to individual centers. When surveying studies examining ECMO in association with cardiac surgery, patients with single-ventricle lesions have not consistently been shown to have poorer survival when compared with patients with 2ventricle lesions. ${ }^{5,13-16}$ However, the ELSO cumulative summary for data collected through 2007 reported survival for infants (age 0-30 days) with hypoplastic left heart syndrome to be $26 \%$ compared with an overall survival of $36 \%$ for infants with any congenital heart disease when supported by ECMO for cardiac failure. ${ }^{17}$ Some discrepancy might be due to differences in classification because some studies have only classified patients with hypoplastic left heart syndrome as a single ventricle, ${ }^{14,15}$ whereas others have classified any patient with intracardiac mixing of systemic and pulmonary blood return as a single ventricle. It is also possible that the subset of patients with cardiac disease undergoing ECPR are different from the larger population of patients with cardiac disease who are started on ECMO for reasons other than cardiac arrest (low cardiac output states, unable to separate from bypass, and respiratory failure) and that key differences in this subset might be the reason behind decreased survival of patients with single-ventricle lesions. 
TABLE 4. Features associated with death in children with cardiac disease undergoing ECPR

\begin{tabular}{|c|c|c|c|}
\hline Variable & Odds ratio & $P$ value & 95\% confidence interval \\
\hline \multicolumn{4}{|l|}{ Pre-ECMO factors } \\
\hline Model 1: Diagnostic classification* $(n=492)$ & & .09 & \\
\hline Two ventricles/pulmonary hypertension & 1.0 & & Reference group \\
\hline One ventricle & 1.6 & & $1.05-2.4$ \\
\hline Muscle disease & 1.2 & & $0.7-1.9$ \\
\hline Pre-ECMO arterial blood $\mathrm{pH}$ & & .01 & \\
\hline $\mathrm{pH}>7.21$ & 1.0 & & Reference group \\
\hline $\mathrm{pH} 7.01-7.21$ & 1.3 & & $0.8-2.1$ \\
\hline $\mathrm{pH}<7.01$ & 2.2 & & $1.3-3.7$ \\
\hline Arterial cannulation site & & .03 & \\
\hline Aorta & 1.0 & & Reference group \\
\hline Right carotid artery & 0.6 & & $0.4-0.9$ \\
\hline Other sites & 1.2 & & $0.6-2.3$ \\
\hline Model 2: RACHS-1 score $\dagger(n=277)$ & & .02 & \\
\hline RACHS 1-2 & 1.0 & & Reference group \\
\hline RACHS 3-5 & 1.3 & & $0.6-2.7$ \\
\hline RACHS 6 & 2.7 & & $1.2-6.2$ \\
\hline Pre-ECMO arterial blood $\mathrm{pH}$ & & .09 & \\
\hline $\mathrm{pH}>7.21$ & 1.0 & & Reference group \\
\hline $\mathrm{pH} 7.01-7.21$ & 1.8 & & $0.9-3.5$ \\
\hline $\mathrm{pH}<7.01$ & 1.9 & & $0.9-4.0$ \\
\hline Arterial cannulation site & & .02 & \\
\hline Aorta & 1.0 & & Reference group \\
\hline Right carotid artery & 0.5 & & $0.3-0.8$ \\
\hline Other sites & 1.3 & & $0.5-3.6$ \\
\hline \multicolumn{4}{|l|}{ ECMO complications $\ddagger(n=485)$} \\
\hline CNS injury on radiology & 3.7 & $<.001$ & $1.8-7.6$ \\
\hline Renal injury & 1.8 & .03 & $1.1-3.1$ \\
\hline Days on ECMO & 1.0 & .04 & $1.0-1.08$ \\
\hline $\mathrm{pH}<7.2$ during ECMO & 3.5 & .001 & $1.6-7.6$ \\
\hline
\end{tabular}

ECPR, Extracorporeal membrane oxygenation as an aid to cardiopulmonary resuscitation; ECMO, extracorporeal membrane oxygenation; $R A C H S$-1, Risk Adjustment for Congenital Heart Surgery-1; $C N S$, central nervous system. ${ }^{*}$ c-statistic $=0.62$. $\dagger \mathrm{c}$-statistic $=0.66$. $\ddagger$ c-statistic $=0.63$.

In our study, patients with cardiomyopathy or myocarditis who were started on ECPR did not have a significantly different rate of survival when compared with postoperative cardiac patients. Additionally, the hospital survival rate of patients with cardiac muscle disease in the present patient population $(45 \%)$ was lower than that in other studies that have examined survival among patients with cardiomyopathy or myocarditis supported with ECMO. In a study by McMahon and coworkers, ${ }^{18}$ children with dilated cardiomyopathy started on ECMO had a hospital survival rate of $89 \%$, with the majority of survivors $(71 \%)$ being bridged to heart transplantation by means of ECMO. Similarly, patients with myocarditis supported with ECMO had a survival rate of $83 \%$, although this did include some patients who underwent a cardiac arrest. ${ }^{19}$ Alsoufi and associates ${ }^{12}$ reported higher survival rates for patients with cardiomyopathy $(50 \%)$ and myocarditis $(67 \%)$ compared with postcardiotomy patients $(36 \%)$ when started on ECMO for ECPR, although these survival rates are still lower than the survival rates of patients with cardiac muscle disease started on ECMO for circulatory support, which might be related in part to organ availability for transplantation within the limited time of ECMO support. The disparity in survival might also stem from having a cardiac arrest. In patients with heart failure and compromised circulation, an arrest event would likely worsen their circulatory status and negatively affect survival. Alternatively, a cardiac arrest in patients with muscle disease might be a sign of rapidly deteriorating circulatory status, and thus these patients might have a poorer clinical status when compared with patients with cardiac muscle disease who have not had a cardiac arrest. Regardless of the underlying cause for this discrepancy in survival rates, supporting patients with cardiomyopathy or myocarditis with ECMO before an arrest appears to be associated with improved survival compared with those treated for arrest.

In our study, a low blood gas $\mathrm{pH}$ was associated with decreased survival. Without the use of ECPR, several factors during cardiac arrest, including prolonged resuscitation times,${ }^{10}$ administration of bicarbonate, ${ }^{2,10}$ and administration 
of high doses of epinephrine, ${ }^{2,10}$ have been associated with decreased survival. Resuscitation time and drugs administered before initiation of ECPR are not recorded in the ELSO database. The blood gas $\mathrm{pH}$ was used as a proxy measure for determining adequacy and duration of CPR because patients with an extreme acidosis likely had inadequate or longer duration of resuscitation before implementation of ECPR. Other studies examining ECPR have produced mixed results regarding an association between length of CPR and hospital survival among patients with heart disease. , $^{4,12,13}$ In fact, the majority of these studies have not found a difference in CPR duration among survivors and nonsurvivors. ${ }^{4,5,12}$ This might suggest that the quality of CPR might be more important than the duration of CPR. It might be for this reason that no upper limit of duration of pre-ECMO CPR for withholding ECPR has been established. Similarly, because $30 \%$ of patients with a blood gas $\mathrm{pH}$ of less than 7.01 still survived to hospital discharge, a lower blood gas $\mathrm{pH}$ limit for withholding ECPR cannot be delineated. However, pre-ECMO arterial blood $\mathrm{pH}$ along with other markers of organ injury might help clinical decision making regarding continued ECMO support. ${ }^{5}$ Blood $\mathrm{pH}$ might also be used as an indirect measure of the pre-ECMO and prearrest circulatory status. From this standpoint, a low blood $\mathrm{pH}$ in a patient who is receiving maximal medical therapy might be an indication for urgent ECMO support before cardiac arrest.

It is notable that right carotid cannulation was associated with increased survival. It is likely that children who were cannulated through the right carotid artery had a closed chest, whereas children cannulated through the aorta likely had an open chest or were in the early postoperative period. ${ }^{5}$ Thus right carotid artery cannulation is probably an indirect index of clinical stability after surgical intervention. Additionally, aortic cannulation can be technically more difficult with more significant complications, such as tamponade and cannula dislodgement. The cannulation site might also be reflective of differences in center practice. ELSO does not release center-related data, and we were unable to adjust for this potential confounder. Finally, children with 2-ventricle lesions and cardiac muscle disease were significantly more likely to be cannulated through the right neck when compared with those with single-ventricle lesions, and although this was included in the analysis, it is possible that this colinearity was not fully accounted for in the logistic regression. The association between neck cannulation and increased survival was not found in a recent report of ECPR use in children with heart disease. ${ }^{12}$ This might be due to a smaller number of patients in their report when compared with our current cohort or possibly because of variability in institutional practice and experience regarding the choice of ECMO cannulation site.

Several ECMO complications, including renal injury, neurologic injury, and persistent acidosis after the initiation of ECMO, were found to be significantly associated with decreased hospital survival. Similar to our findings, other stud- ies consistently report that renal injury, $5,13,15,16,20,21$ neurologic injury, ${ }^{15}$ and increasing time on $\mathrm{ECMO}^{13,16}$ are associated with decreased survival. Use of renal replacement therapy was not independently associated with increased mortality likely caused by colinearity with renal injury. In a study by Huang and colleagues, ${ }^{16}$ higher lactate levels during ECMO were associated with decreased survival. Although the ELSO database does not report lactate levels, persistent acidosis during ECMO was associated with decreased survival in our study. This suggests that persistent insufficient circulatory support on ECMO is a poor prognostic factor. In this current cohort, hepatic failure, as evidenced by hyperbilirubinemia, tended to be associated with decreased survival. Although not as prevalent as renal injury in other studies, liver failure has also been described as a risk factor for poor hospital survival. ${ }^{5}$ Other factors that had been previously associated with decreased survival but not confirmed in this study included serious circuit complications, ${ }^{20}$ age, and male sex..$^{5}$

There are several limitations of this study. Although the definition of ECPR has been delineated and generally accepted as receiving chest compressions while being cannulated for ECMO, the classification of ECPR is dependent on each institution's voluntary submission of data and coding accuracy. Similarly, the accuracy of information regarding diagnosis and procedures is dependent on the accuracy of each institution. The ELSO registry provides definitions for ICD-9 codes, as well as CPT codes, in the forms provided to ELSO members. ${ }^{22}$ Although ELSO does conduct audits by asking institutions to resubmit information for a sample of patients, it does not independently verify submitted information. It is only recently that ELSO began collecting information regarding operative details of patients undergoing cardiac surgery. This information is currently not available for analysis and limits information regarding surgical intervention and timing of ECMO support. The ELSO database contains all operative procedures that a patient had during the hospitalization when he or she underwent ECMO but not when these procedures occurred. No details of the temporal relationship between each operation and the patient's ECMO run are available. When assigning RACHS-1 scores, the highest RACHS-1 score assignable was given with the assumption that the patient was started on ECMO after the most complicated procedure. It is possible that patients undergoing cardiac surgery could arrest and be placed on ECMO before this surgical procedure. With a few exceptions, it is more likely that patients would arrest after heart surgery rather than before a procedure. There is also the possibility of misclassification bias caused by the heterogeneity of the diagnoses listed for each patient. Some patients in the ELSO database had multiple diagnoses that included cardiac disease among others. Although one would expect patients with congenital heart disease to arrest for cardiac reasons, it is possible for these patients to arrest as a result of other causes, such as 
respiratory disease, toxic ingestions, or sepsis. Using the current ELSO database, it is not possible to discriminate between concurrent illnesses and the immediate cause of an arrest.

\section{Conclusion}

We found that ECMO used as a rescue therapy for CPR was associated with survival in more than $40 \%$ of patients who faced a high likelihood of death without mechanical support. In this group single-ventricle physiology or those undergoing more complex cardiac surgery had decreased odds of survival. Additionally, pre-ECMO acidosis was also associated with decreased survival, stressing the importance of adequate CPR and rapid deployment of ECPR, as well as consideration of using ECMO before an arrest in a clinically deteriorating patient. Finally, evidence of renal injury, central nervous system injury, and persistence of inadequate systemic perfusion during ECMO were predictors of poor survival once a patient was started on ECMO.

\section{References}

1. Perondi M, Reis A, Paiva E, Nadkarni V, Berg R. A comparison of highdose and standard-dose epinephrine in children with cardiac arrest. $N$ Engl J Med. 2004;350:1722-30.

2. Rhodes J, Blaufox A, Seiden H, Asnes J, Gross R, Rhodes J, et al. Cardiac arrest in infants after congenital heart surgery. Circulation. 1999; 100:II194-9.

3. del Nido PJ, Dalton HJ, Thompson AE, Siewers RD. Extracorporeal membrane oxygenator rescue in children during cardiac arrest after cardiac surgery. Circulation. 1992;86:II300-4.

4. Morris M, Wernovsky G, Nadkarni V. Survival outcomes after extracorporeal cardiopulmonary resuscitation instituted during active chest compressions following refractory in-hospital pediatric cardiac arrest. Pediatr Crit Care Med. 2004;5:440-6.

5. Morris M, Ittenbach R, Godinez R, Portnoy J, Tabbutt S, Hanna B, et al. Risk factors for mortality in 137 pediatric cardiac intensive care unit patients managed with extracorporeal membrane oxygenation. Crit Care Med. 2004;32:1061-9.

6. Thiagarajan R, Laussen P, Rycus P, Bartlett R, Bratton S. Extracorporeal membrane oxygenation to aid cardiopulmonary resuscitation in infants and children. Circulation. 2007;116:1693-700.
7. Available at: http://icd9cm.chrisendres.com/. Accessed Aug 24, 2007.

8. Available at: https://catalog.ama-assn.org/Catalog/cpt/cpt_search.jsp? checkXwho=done. Accessed Aug 24, 2007.

9. Jenkins K, Gauvreau K, Newburger J, Spray T, Moller J, Iezzoni L. Consensus-based method for risk adjustment for surgery for congenital heart disease. J Thorac Cardiovasc Surg. 2002;123:110-8.

10. Reis A, Nadkarni V, Perondi M, Grisi S, Berg R. A prospective investigation into the epidemiology of in-hospital pediatric cardiopulmonary resuscitation using the international Utstein reporting style. Pediatrics. 2002;109:200-9.

11. Parra D, Totapally B, Zahn E, Jacobs J, Aldousany A, Burke R, et al. Outcome of cardiopulmonary resuscitation in a pediatric cardiac intensive care unit. Crit Care Med. 2000;28:3296-300.

12. Alsoufi B, Al-Radi O, Nazer R, Gruenwald C, Foreman C, Williams W, et al. Survival outcomes after rescue extracorporeal cardiopulmonary resuscitation in pediatric patients with refractory cardiac arrest. $J$ Thorac Cardiovasc Surg. 2007;134:952-9.

13. Aharon AS, Drinkwater DC Jr, Churchwell KB, Quisling SV, Reddy VS, Taylor M, et al. Extracorporeal membrane oxygenation in children after repair of congenital cardiac lesions. Ann Thorac Surg. 2001;72:2095-101.

14. Hintz S, Benitz W, Colby C, Sheehan A, Rycus P, Van Meurs K, et al. Utilization and outcomes of neonatal cardiac extracorporeal life support: 1996-2000. Pediatr Crit Care Med. 2005;6:33-8.

15. Baslaim G, Bashore J, Al-Malki F, Jamjoom A. Can the outcome of pediatric extracorporeal membrane oxygenation after cardiac surgery be predicted? Ann Thorac Cardiovasc Surg. 2006;12:21-7.

16. Huang S, Wu E, Chen Y, Chang C, Chiu I, Chi N, et al. Experience with extracorporeal life support in pediatric patients after cardiac surgery. ASAIO J. 2005;51:517-21.

17. ECMO Registry of the Extracorporeal Life Support Organization. Ann Arbor (MI): Extracorporeal Life Support Organization; 2007.

18. McMahon A, van Doorn C, Burch M, Whitmore P, Neligan S, Rees P, et al. Improved early outcome for end-stage dilated cardiomyopathy in children. J Thorac Cardiovasc Surg. 2003;126:1781-7.

19. Duncan B, Bohn D, Atz A, French J, Laussen P, Wessel D. Mechanical circulatory support for the treatment of children with acute fulminant myocarditis. J Thorac Cardiovasc Surg. 2001;122:440-8.

20. Chaturvedi R, Macrae D, Brown K, Schindler M, Smith E, Davis K, et al. Cardiac ECMO for biventricular hearts after paediatric open heart surgery. Heart. 2004;90:545-51.

21. Hoskote A, Bohn D, Gruenwald C, Edgell D, Cai S, Adatia I, et al. Extracorporeal life support after staged palliation of a functional single ventricle: subsequent morbidity and survival. $J$ Thorac Cardiovasc Surg. 2006;131:1114-21.

22. ELSO Registry Form Equipment Lists and Supporting Documents. Available at: http://www.elso.med.umich.edu/support.htm. Accessed January 29, 2008. 